\title{
Health Care Expert's Readiness to Implement National Unified Medical Records (Numr) System in the United Arab Emirates; A Qualitative Study
}

Alya Harbi ( $\sim$ alyaharbi@outlook.com )

British University in Dubai; Director, Statistics and Research Centre, Ministry of Health and Prevention,Dubai, United Arab Emirates.

\section{Research}

Keywords: Readiness, electronic medical records, National Unified Medical Records, NUMR, centralized information system, United Arab Emirates

Posted Date: August 20th, 2020

DOl: https://doi.org/10.21203/rs.3.rs-61668/v1

License: () (1) This work is licensed under a Creative Commons Attribution 4.0 International License. Read Full License

Version of Record: A version of this preprint was published at Informatica on July 10th, 2021. See the published version at https://doi.org/10.31449/inf.v45i5.3358. 


\section{Abstract \\ Background}

Electronic health records provide a valuable resource to improve health evaluation, surveillance and informs effective clinical decision making. Although electronic medical records (EMR) are introduced in many different settings, yet little is known about the readiness, effectiveness and progress of a centralized information system like NUMR across United Arab Emirates. This study aims to determine the readiness for national unified medical record (NUMR), barriers and challenges from the point of views of experts working in the field of health informatics on daily basis.

\section{Methods}

Semi-structured interviews were conducted with 10 experts using purposive sampling method from different healthcare organizations in the United Arab Emirates. Perceptions of interviewees regarding the readiness for national unified medical record were elicited. Thematic analysis was used to identify the different themes extracted from the interview transcripts.

\section{Results}

Overall, seven themes emerged after analyzing the transcripts obtained from the respondents. This study is expected to bring countless benefits for all countries that are moving forward toward nationwide and interoperable healthcare platforms. The support for NUMR will make a significant contribution to the health systems across UAE that will ultimately lead to safety, access to single shared records, improved care and coordination, better clinical and decision making, faster engagement, easy and secure access, safety and will save time for other tasks.

\section{Conclusion}

The future of implementation of NUMR in UAE is promising. Our findings offer beneficial guidelines for consideration in implementing NUMR system across UAE and also helps in the drive to improve healthcare systems nationally.

\section{Introduction}

With the advancement in the information communication technologies, field of interoperability, and health data exchange in the past era, a diversity of the systems are applied across different healthcare institutions to improve the healthcare services with improved decision making, communication and data management. Of these, implementation of the National Unified Medical record (NUMR) across UAE is the priority agenda not solely in the developed nations but also in several other developing countries. ${ }^{1}$ Federal Law No. 2 of 
2019 issued by UAE federal government on the utilization of Information and Communication Technology (ICT) in health field aims to introduce the establishment of central system as well as regulate the collection, processing, and transfer of electronic health data originating in the UAE. Such a system if implemented is expected to improve the quality of healthcare by means of reducing medical errors, improves work flow, increases revenues, minimize treatment time and cost, improving the care of patients by forming a better linkage for all care givers, limiting the need for workers, supplies and space for filing or retrieval of medical records. ${ }^{2}$ Several healthcare organizations from across the globe have applied EHR systems for the purpose of improving the process to record information but only a few have been so far successful. ${ }^{3}$ The total percentage of breakdowns and failure are disturbingly higher in meeting the desired goals after its implementation and adoption. More than fifty percent EHR systems globally either fail or fail to be used appropriately. ${ }^{4,5}$ Opposition and resistance in shifting from the paper-based systems to electronic systems may generate some other concerns. These may include the lack of organizational readiness ${ }^{6,7}$, lack of preimplementation activities, funding and lack of technical or computer skills of personnel and unavailability of technology. ${ }^{8}$

Only limited studies have been reported or conducted on the readiness of healthcare professionals and experts before actually implementing the actual system. A manual has also been created by the World Health Organization (WHO) that outlined the preliminary concerns such as health professional's readiness, infrastructural, technical, and organizational readiness for a new work practice with respect to the electronic healthcare records. ${ }^{9}$ Majority of the pre-implementation assessments often failed to take in the knowledge and attitude (readiness) of experts that may help in determining the success factors for implementing the NUMR system across UAE.

Thus, the readiness assessment conducted is a part of pre-implementation assessment which is important and also comprises of the human factor. ${ }^{10}$ It helps in examining the preparedness of each organizational component for implementing new system. Thus, this will also help in identifying the focus areas that needs to be done during implementation. As mentioned in the previous studies, one primary barriers to implement the NUMR successfully is whether the clinician accept the potential disruptions, new systems and changes that follow. ${ }^{11,12}$ It has been indicated that health workers perceived NUMR as interfering with the clinical workflow, introduces disruptive changes to workplace, and reduces productivity. Such a tendency is also serious in developing countries where the anxiety related to computers are higher. ${ }^{13}$

Existing evidence mainly provides a cursory examination of the electronic health record implementation. However, a deeper and updated examination of the challenges, usage of NUMR, and set of standards to follow does not exist. Thus, the future plans for implementation and current readiness assessment are still left untapped. This study will therefore guide in filling these gaps in the existing body of evidence as it will determine the perceptions and experiences of the experts who use the EHR systems on a regular basis. The findings of this study will serve United Arab Emirate's hospitals, health professionals, and expert's important evidence to plan and make decisions accordingly. The study aims at determining the benefits, how and whys of the NUMR implementation. This study also delves into how e-Health standards are being 
utilized, readiness for national unified medical record, barriers and challenges from the point of views of experts working in the field of health informatics on daily basis.

\section{Methods}

\section{Research Design/Settings}

A qualitative study was conducted to purposively collect data from 10 experts from different healthcare organizations in the UAE such as Department of Health, Abu Dhabi, Ministry of Health and Prevention, Dubai Health Authority, Cerner Middle East and Sharjah university hospital in the UAE.

\section{Participants}

A purposive sampling method was used to recruit 10 experts and vendors from different healthcare organizations involved in the planning for national unified medical record in the UAE. The participants were interviewed until data saturation was reached.

\section{Data Collection Procedures and Tools}

Semi-structured in-depth interviews are performed to study the individual perceptions and viewpoints. This type of data is gathered directly from the personals or from the real life experiences of the respondents. The open-ended question in the interviews not only helps to study the respondent's opinions but it also helps the researcher to establish, a strong relationship with the respondents during the interview that helps in data provision. Around 10 experts in the field of NUMR application were interviewed. In total, 12 questions were developed which mainly covered information about the readiness for National Unified Medical Record (NUMR) across UAE along with the standards and methodology currently being followed towards establishing NUMR to support the provision of high-quality healthcare. The interview instrument also included questions about the current capacities and data system being used, informatics capacity, barriers and challenges, and future recommendations to overcome these shortfalls. These interviews were conducted by the investigators that lasted for an average of 10 minutes. Each interview was verified, transcribed, recorded and coded thematically.

\section{Data Analysis}

Thematic analysis was used for evaluating the data. This is an extensively used method for analysing any qualitative research. Braun and Clarke ${ }^{14}$ described how to use thematic analysis in a step-by-step way. Thematic analysis is a foundational analysis method that is needed to be described or defined for solidifying its place in a qualitative research. Thematic analysis is easy to use method that helps investigators who are unaware of other most intricate categories of qualitative investigations.

\section{Ethical Approval}

Ethical approval was sought and granted by two organizations. One from the university board of the British University in Dubai and another approval was taken from the Research and Ethical (REC) committee from 
Ministry and Health and Prevention by applying to them and filling their ethical approval form. The privacy and confidentiality of the interviewees was also maintained. Informed consent was also obtained.

\section{Results}

The study aims to comprehend the reality from the standpoint of the experts involved in that specific situation or environment. We interviewed the experts from different healthcare organizations regarding the readiness for National Unified Medical Record (NUMR) across UAE along with the standards and methodology currently being followed towards establishing NUMR to support the provision of high-quality healthcare.

\section{EMR systems/vendors vs. paper-based system and its utilization in UAE}

In this category, the interview data focusing on the existing use of EMR was analysed. The statements of the interviewees' regarding the EMR systems that are being used in both public and private hospitals were discussed. Majority of the respondents avowed that Cerner is being used, and viewed by several independent entities including insurance companies, public hospitals, private hospitals and primary care physicians in Dubai, Abu-Dhabi and Northern Emirates. As one of the participants stated;

"In both the outpatient and inpatient settings, Cerner and Epic are the top choices, while vendors such as eClinicalWorks,Allscripts,Meditec, Health Insights are trailing much further behind" (P5)

A participant from the federal government stated:

"Cerner is used in public and other systems in private hospitals like Trakcare" (P8)

Another vendor added:

"In Dubai, we use Epic system in public sector that runs in 4 hospitals \& 23 clinics; whereas, in private sectors, 32 private hospitals in Dubai uses many other systems such as Cerner, Meditec, and Sage etc." (P4).

A participant from SEHA-Abu-Dhabi indicated that:

"At SEHA, all hospitals are utilizing an electronic medical record (Cerner)." (P9)

Moreover, participants also mentioned that electronic medical records are being increasingly used in almost every private and public hospitals to access and document the patient records along with online decision-making tools, medical information as well as prescribed medications. They have also altered the dynamics of the patient-clinician interaction through telemedicine, virtual consultations and clinician patient email. The participants also agreed on the fact that an EMR system helps to promote the development of health care transactions from a wasteful, paper-based system to a real-time, more reliable, non-paper-based system. The charge of transcription, manually taking of notes, the dictation time, and the 
writing of medical prescriptions are practically eradicated. It allows the doctor to be more competent as well as it helps to propose better services to more patients. For example, one of the participants stated that:

"All of them have a sort of system in place. EMR improves quality of care and increases levels of client satisfaction. It also reduces the use of paper, which has an impact on hospital expenses" (P5)

On the contrary, another participant from Dubai Health Authority reported that:

"Only small to medium healthcare provider facilities are partially manual in Dubai" (P4)

A participant from Sharjah added:

"Mainly most of the other hospitals are using electronic medical records but not totally used some are still dealing with papers especially with consent forms (both public and private)." (P7)

Hence, these responses sufficiently confirm that EMR systems are now much better, vibrant, embedded fully in the hospitals culture, and constitutes the core of a computerized health care system to promote greater quality and efficacy in the health care delivery.

\section{Medical Devices Integration (MDI)}

Some of the participants stressed upon the significance of Medical Devices Integration (MDI) solution that helps to establish a connection to transfer data between an information system and a medical device. Middle solutions are standard operating systems for Electronic health records to finally end the lack of interoperability along with bringing disruptive transformation, driving change, and innovation to solve EHR interoperability challenges.

One of the participants stated:

"MDIs and other medical devices in most of the facilities are all connected and seamlessly integrated to the EMR through the iBus solution. Cerner CareAware iBus solution will enable you to connect medical devices to the patient's EMR, enabling patients and caregivers to access the right information at the right time."

On the contrary, other participants reported some other devices currently being used in their facilities. For example, one of the participants revealed that:

"DHA is using Orion Rhapsody as an MDI solution to integrate EPIC with other" (P3)

Another participant added that multiple solutions are being used:

"There are multiple solutions that DHA is using as Middleware during the EPIC implementation." (P4)

"All Lab devices are connected to Cerner EMR." (P6) 
"SEHA has the full complement of Cerner solutions for device connectivity and integration into the EMR" (P10)

These responses indicates that Rhapsody is a healthcare-focused interoperability platform that is being used by the health systems, public and private hospitals, vendors, health information exchanges, public health departments and while, open link is being used by federal government organizations that mainly helps to enhance healthcare ecosystems through seamless connectivity for the purpose of unlocking the potential of data, on-premises as well as in the cloud, paving the way towards a healthier society.

\section{Other Clinical Information Systems}

There are three ancillary systems that are the base of any electronic health records. These include pharmacy, radiology and laboratory systems. To capitalize fully, it is important to have these ancillary systems to be fully integrated or centralized across the integrated delivery system. Our results suggest that many providers have chosen ancillary systems being offered by their existing vendors to avoid the need for potentially costly and complex interfaces. As one of the participants stated:

"Different systems have been used at DHA and were integrated with EPIC during the implementation. For example, DHA uses AGFA Radiology Enterprise Solutions for PACS and Diagnostics Management." (P4)

Another participant from Dubai added:

"Pharmacy: Epic Willow; Lab Information system: SunQuest; Radiology: Agfa" (P5)

A participant from Sharjah added:

"Pharmacy System is part of TrakCare system, and it includes inpatients and outpatient modules and integrated with Insurance systems of electronic approval." (P7)

Our results suggest that most of UAE hospitals have transformed beyond stage 2 of the EMR Adoption Model. The above results also highlight that majority of the hospitals do have all 3 ancillary systems (radiology, laboratory, and pharmacy) installed.

\section{Readiness for National Unified Medical Record}

Respondents were further inquired about the state of organization's readiness for National Unified Medical Record in UAE to explain why EMR initiatives may fail or succeed in future. Determining readiness of an organization is an initial step that a person needs to take in order to assure that EMR is being fully utilized. Most of the respondent agreed that the readiness level of UAE for national unified medical record is high because of several reasons like government sponsorship, availability of regulations and standards, compliance to the interoperability standards, availability of the hospital level EMRs that comply with international standards, infrastructure maturity as well as smart devices and smart services penetration. The participants unanimously stated, 
"Yes! UAE and especially the emirate of Dubai is ready for implementing HIE due to the digital readiness of hospitals and the high demand for connected care to increase efficiency and improve quality of care." (P3)

A participant from Sharjah quoted the example from Abu-Dhabi and added:

"Yes, because most of UAE hospitals are using the different electronic system and they are satisfied in this experience especially with government hospitals and moving to a unified medical record will access the government and the patient for better access and better communication system. It is challenging project as some providers are still using manual medical records, I feel such projects can go in stages, taking an example Abu Dhabi that has launched Malaffi project to unify the medical record in Abu Dhabi Emirate." (P7)

While a participant from Abu-Dhabi clarified that:

"Not all public and private hospitals in Abu Dhabi emirate have moved to an electronic medical record system. Although with the upcoming implementation of Malaffi HIE there will be the need for all public and private hospitals and clinics to interface to the Patient Portal and Health Information Exchange. This will likely take several years to be fully realized but should get closer to a situation where all vital information for patients in Abu Dhabi will have some type of medical based information in the HIE system." (P10)

Another respondent stated that a national policy on unified electronic medical record should be established to help its implementation. As mentioned below:

"Yes, the UAE is ready for NUMR. The main Public Health Providers in the UAE (i.e. SEHA, DHA and MOHAP) have implemented EMR solutions in their digital transformation journey. It is important that NUMR ensure vendor agnostic approach without any EMR monopoly. Also, the private sector needs to be allowed to choose their EMR according to their budgets and capabilities. The regulators will need to understand and implement a framework to certify Healthcare IT Applications, EMRs, Informatics solutions and other HIT systems." (P4)

Interestingly, all participants confirmed that UAE will succeed in the system integration and improve interoperability. UAE will be capable to accumulate a large amount of health data for the entire patient population. Moreover, patients will be more apt to become involved actively in managing health in addition to taking part in shared decision making because of having easier access to their health information. Furthermore, the utilization of the electronic medical records can make office of physicians more efficient and it may also improve the quality care of the patients by making their medical history available for any physicians who are treating them. As one of the participants stated:

"The UAE is ready for the National Unified Medical record. This is becoming essential to control the population health and drive clinical programs with public/private sector to improve the health of the population, control the registries managing chronic diseases and predicting health outcomes." (P6)

Upon digging deeper within the interviews and the argument on the perception of respondents about the readiness for NUMR, it was found that, Unified electronic health records were strongly supported by most of 
the respondents:

"A stronger system of health IT can often increase communication between patients and providers as well as may assist to foster enhanced patient engagement through the use of applications such as patient portals and interfaces with laboratory, radiology, and medical devices." (P1)

Moreover, formalized plans should be established with detailed strategies for effective implementation of NUMR.

\section{EMRAM assessment by HIMSS}

EMRAM also known as "Electronic Medical Record Adoption Model" is a unique evaluation model and a strategic roadmap for effective EMR maturity and adoption developed by HIMSS Analytics. These 8 staged models aid to measure the utilisation and adoption of EMR functions needed to attain a paperless environment that harnesses technology for optimizing patient care along with enabling benchmarking and comparison with peers. Majority of the respondent addressed the use of EMRAM in their facilities. One of the participants from the Ministry of Health and prevention stated:

"MOHAP performed HIMSS EMRAM assessment in two hospitals, they both got level 6 . There is a plan to perform the same assessment for the rest of the hospitals. EMRAM allows to track progress against other healthcare organizations across the globe." (P2)

While, another respondent from DHA specified:

"Yes, DHA's facilities, including Dubai and Rashid Hospital as well as 12 DHA primary healthcare facilities etched their mark on the hospital digital roadmap by obtaining EMRAM level 6 certification. DHA's primary healthcare centres that received HIMSS Level 6 include Al Badaa, Al Barsha, Al Khawaneej, Al Lusaily, Al Mamzar, Al Mankhool, Al Mizhar, Al Towar Al Safa, Nad Al Hamar, Nad Al Sheba Health Center and Za'abeel health centre" (P4)

On the contrary, another respondent who witnessed a survey in Dubai stated:

"The last Dubai EMRAM survey was conducted in 2017 with a 100\% participation of Dubai public and private hospitals. 33 hospitals participated in the survey in which $48.5 \%$ of them got stage 4 or above." (P3)

A participant from Sharjah added:

"Our Hospital UHS is HIMSS Stage 6." (P7)

These responses confirm that UAE continues to uphold its position as it has some of the most digitally mature healthcare organization, after magnificently accomplishing stage 6 level of the HIMSS Analytics Electronic Medical Record Adoption Model (EMRAM).

Recommended standards, methodology, models and system architecture 
As soon as the third-party applications that were being utilized within EHRs increased in the past, there emerged a need to establish specific and additional interfaces. Therefore, it soon became apparent that standards were required. IEEE P1157 and Health Level Seven (HL7) were considered as the foremost interface standards internationally by the homegrown vendors or EHRs to interface with several different systems. The benefit of such a strategy was to lessen the ambiguity in definitions of the data element. As soon as the EHRs number and other applications started to be interfaced, the HL7 standard was expanded in addition of being refined into supplementary domains to turn into a most practical solution for aggregating ancillary systems like echocardiography, electrocardiogram, microbiology, laboratory, and other outcomes into a central EHR. Based on the participant response, most of them affirmed using HL7 and FHIR:

"There are international standards that could be customized in the UAE. HL7 and FHIR are important Health Information Exchange protocols that could be adopted. For an example, please visit the interoperability standards that was develop at DHA." (P4)

The key focus of EMR systems is the secure, efficient storage and data retrieval. The government wanted to be sure that EMR systems are secure, the privacy of users is being maintained, and data is shared only between departments that are authorized. For achieving this, the federal government has established a set of standards for the system of EMR. As one of the participants stated:

"FHIR, CDA and LOINC. SNOMED can also be considered in HIE should capitalize on existing standards put forward by eclaims system e.g. ICD10, CPT4 and Pharmacy RX and promote the usage of HL7 in future $(P 3)^{\prime \prime}$

These interview results confirm that to hasten the deployment of EMR, one should focus on the interfaces rather than the EMR system. We often possess the interface solutions in the form of standards: HL7/ASTM, IP, DICOM, LOINC, SNOMED, and others developed by the community of medical informatics. One may only need to embrace them.

Respondent were further inquired about the models and system architecture recommended for the project. Majority of the participants agreed that FHIR resources (collection of information models) decisively fit within the domain of information architecture whereas, the FHIR APIs for data exchange address aspects of application architecture. As stated by one of the participants:

"Due to the federal/local setup of healthcare authorities in the UAE and federated architecture is the best option where every local regulator can govern data sharing in its jurisdiction and then exchange patient record throughout the regulator nodes. Open architecture must be adopted and FHIR web services should be the main approach for data exchange. (P3)"

Another participant further indicated that API based predictive modelling services is easy to implement across thin client applications, particularly in the mobile environment. FHIR outlines resources characterized as XML or JSON objects that often comprise of health ideas in addition to reference and 
searchable parameters. FHIR additionally defines RESTful API URL patterns for reading, creating, updating, and deleting (CRUD) operations.

"Web services through the FHIR standard as this architecture is standard and all EMR should be conforming to this healthcare integration API's." (P6)

Another respondent from the Ministry of Health and Prevention added;

"From operational point of view, the National unified medical record will be in partnership with the private sector on a PPP model (Public Private Partnership). The technical architecture will be hybrid (not fully centralized and not fully fragmented), to accommodate the local requirements of the local authorities as well as the federal requirements. Integration and communication will be using different standards like HL7, web services, XML, SOAP, DICOM, etc." (P2)

A participant from SEHA stated:

"Follow the HIMSS model, JCIA and JAWDA requirements and this should encompass 95\% of what is needed to accomplish the project and be certified or ready for certification on its deployment." (P10)

In terms of system architecture, majority of the participant stated that a distributed electronic healthcare system founded on the service-oriented architecture (SOA) can help developers to integrate several different kinds of databases, software applications, and computing platforms within a certain health network along with state, community, and national health information exchanges.

"SOA will be suiting such project, managing technology dependency is required in such projects, and the load tolerance is a major aspect in such a project due to the high volume of transaction." (P7)

"SOA should be used" (P8)

\section{Addressing Challenges to Optimal Use and implementation of NUMR}

Different government agencies and health organizations often recognize the values of information in electronic health records to examine the optimal care patterns. Staff (resistance, lack of training), costs, and concerns related to the technology (interoperability, issues during implementation, security and privacy) were among the most frequently mentioned barriers. IT issues for example, implementation not being available for all information systems and internet not reliable can exasperate an already stimulating condition.

One of the questions asked was about the concerns of the respondents with respect to the privacy and confidentiality of the electronic medical records. The results found out that the participants were concerned about it in general and some emerging issues facing privacy, healthcare coverage, and especially the EHR security remains to be a critical challenge for its acceptance. All participants stated their concerns for the security of personal health and patients' safety concerns. One respondent stated: 
"Some of the risk and challenges may include Information security and documentation completeness. Moreover, Data privacy and ownership became common issues whenever data exchange and storage through computer networks were planned" (P1)

In response to addressing further risk and challenges that may be faced while implementing this project, one of the respondents from the Ministry of Health and Prevention stated:

"There are few risks and mitigation actions identified for these risks such as Sustainability, that's why PPP operating model is selected, adoption, private sector maturity, alignment between federal and local authorities, data quality, privacy and security, stakeholder's engagement, and marketing and awareness" $(P 2)$

Another respondent from the same organization added;

"Confidentiality, security, completeness, timing and validity of data" (P8)

These findings are consistent with Bramble et al. (2010) who argued that larger practices tend to have access to the potentially greater resources (human resources and financial) that is needed for unified health system adoption and delivery as well as it must include extensive training and internal IT assistance.

A proper structure for governance needs to be put in place for achieving transparency, independence with respect to decision making and other objectives. One of the participants indicated that for successful exchange of data to be possible, healthcare professional should follow similar documentation and information governance processes. As stated below:

"Governance will be important. A unified body of experts to make decisions that impact the course and direction of the project will be imperative. There must be senior cabinet level government officials that are helping support and drive the initiative and require and mandate what needs to be done. Otherwise, I fear there will be too many ways to interpret how the system should be used, causing confusion, mistrust and eventually non-use of the system." (P10)

Thus, providers/ leadership buy-in and employee leading to resistance and hesitancy, resources, security and privacy, workforce, funding, and lack of technical guidance are some of the experienced challenges that keeps many organizations away from the benefits of NUMR mentioned above. The challenges for the health organizations have been too great to move beyond, although efforts are being made towards this goal.

\section{Discussion}

Our results supplied supportive quotes and themes emerging from the viewpoints of experts belonging to different healthcare organizations. The themes covered topic such as the readiness for National Unified 
Medical Record (NUMR) across UAE along with relevant standards and methodology currently being followed towards establishing NUMR to support the provision of high-quality healthcare.

The current EMR system in seven emirates of UAE were scrutinized through interviews to compare the levels on the EMRAM assessment by HIMSS. ${ }^{15}$ Thereby, this study helped us to identify the barriers towards the implementation of unified use of EMR data in different regions of the United Arab Emirates that can be used to inform future unified electronic medical records. El-Hassan et al ${ }^{16}$ suggested that the findings from Dubai EMRAM has demonstrated that a substantial number of hospitals across the emirates are considerably making a good progress with their journey for automation. This therefore, highlights the educational value for EMRAM that raises awareness with respect to the implementation of EMR as well as fosters support for e-health capacity building.

Factors that affect the healthcare organisations readiness to implement interoperable information systems have also been studied in the past. ${ }^{17,18}$ Nevertheless, most of the respondents in our study indicated that UAE is ready for the National Unified Medical record. This is because it is important to control the health of the population and to drive clinical programs with private/ public sector to improve the health of the population, control the registries, and manage chronic diseases along with predicting the health outcomes. Still adequate investment is required to get the EMR established alongside trying to keep it running. Zandieh and colleagues ${ }^{19}$ revealed that health care institutions with successful implementation of EMR had made sufficient financial investment before adopting EMR for which funds were also maintained. ${ }^{8}$ While in some other countries such as Australia and United States, funds have been allotted for EMR adoption at national level. ${ }^{20,21}$

For exchanging information fluidly, it is important to adopt standards on which the health care systems coincide. Hence, healthcare organizations that seeks to unify criteria in agreement of interoperability such as HIMSS and HL7 International emerged. To exchange a common set of data elements, some of the most commonly used terminology includes "LOINC and SNOMED", common data structures, and a common transport standard, "the HL7 Clinical Document Architecture (CDA) and The American Society for Testing and Materials (ASTM International) Continuity of Care Record (CCR)" are utilized. ${ }^{22}$

Our results suggest that HL7 (Health Level Seven, 1994) is thought to be a standard of choice for communicating clinical information for example, referrals, notes, diagnostics results, clinical trials data, problems, nursing notes, scheduling information, master file records, and more. HL7 offers the structure such as set of database records for exchanging the information of the patients between source systems like dictation, pharmacy, and laboratory systems data repositories for example, medical record systems, performance databases and cancer registries. The HL7 offers all its proposals, minutes and draft standards on the internet without any cost. Other standards reported in our study included FHIR, IHE, CDA, LOINC, and SNOMED.

A strong governance model is crucial to optimize cross functional alignment, to assist in enforcing accountability and to escalate concerns with respect to appropriate decision makers. ${ }^{23}$ The measurable advantages for NUMR needs an effective structure for governance to ascertain that it remains fit for 
purpose as the program evolves. A successful model of governance must comprise of a broad representation from the UAE healthcare ecosystem as to ensure fair and balanced representation of views on a specific subject matter and to bring together context, relevance and needs of all the UAE. ${ }^{16}$ The structure for governance shall offer a balance between tactical and strategic focus for achieving short and long term NUMR business goals. Hence, for this reason, the strategic level will possess a view in long term while the operational level will translate such goals to accountable and practical steps. ${ }^{17}$ Five critical challenges and barriers identified included: (1) funding; (2) resource provision; (3) commitment and support; (4) no control over decisions, (5) lack of training.

The findings of the study were consistent with $\mathrm{Rae}^{23}$ where it was stated that Abu Dhabi is very mature in health informatics especially that DOH has established unified medical records across all Abu Dhabi which is called Malaffi system. It has made a progress towards centralized electronic record. The study conducted by Drew ${ }^{25}$ referred to the implementation of MEGAHIT that can actually be utilized in UAE. Presently, the system of healthcare in the United Arab Emirates is facing a range of financial, demographic, and capacity challenges to provide high quality healthcare services to the UAE population. ${ }^{26}$ Given the potential advantages of NUMR that can bring to address these challenges by means of reducing the inefficiencies and expanding the system catchment making more value for less spends. Hence, this study demonstrates how implementation of NUMR across the United Arab Emirates is essential and how it become even more crucial over time.

One of the strengths for this study is that the data being gathered qualitatively is based on the rich data from interview conducted from 10 experts who were directly leading or working with the informatics systems on a routine basis. While the limitation of this study includes interview fatigue, self-reported, unverified information, and time constraints. The responses may vary and may lead to data bias towards some issues versus underlying concerns. Hesitancy to speak on behalf of an organization's health informatics may have resulted in a limited view of the concerns. The interviews were however, conducted with the staff who were either leading the department or working with health informatics. Lack of full understanding with respect to the systems being used was a barrier to getting a full knowledge and view of these issues. Lastly, this study also includes limitations of the self-reported data that have not been validated or verified independently. Despite of all these limitations, the information obtained, and outcomes are adequate to generalize feelings and readiness of the respondents on the EMR systems being used.

\section{Conclusion}

The progress and capacity of NUMR systems is far-reaching and effective. This study evaluated the expert's perceptions on the readiness of UAE population to adopt NUMR. The quality of care is expected to improve significantly with the introduction of NUMR system and patients waiting time in the hospital is also expected to reduce in general. The findings showed that all the experts and managers recruited for the interviews from different health organizations recognized the importance of implementing NUMR. This support will therefore make a significant contribution to the health systems across UAE that will ultimately lead to safety, access to single shared records, improved care and coordination, better clinical and decision 
making, faster engagement, easy and secure access, safety as well as will save time for other tasks. Some of the organization affirmed using HL7 - FHIR (Fast Healthcare Interoperability Resources) standards. Additionally, SOA tools helps to reduce costs along with minimizing complexity by using a flexible, secure, and standards-based middleware platform. Based on the data gathered from the governmental reports published in the United Arab Emirates, some of the standards being used across the healthcare facilities of the UAE include CPT, ICD-10 CM, RxNorm, HL7V2.x, and DICOM. While, to a large extent, UAE has already adopted ICD10, SNOMED, LOINC, CPT, RxNorm fdb MEDk. The JCl accreditation standards shall be utilized to modulate the management of core functions of the NUMR project such as including cost, time, scope and quality. Management of healthcare workflow and organizational culture can be accomplished through establishing a strategic model founded on standardization, project management, training, governance or controlling leadership. The continuation of these standards across the country is also recommended in future.

\section{Abbreviations}

National Unified Medical Record (NUMR); Information and Communication Technology (ICT); World Health Organization (WHO); Research and Ethical Committee (REC); Electronic medical records (EMR).

\section{Declarations}

\section{Availability of data and materials}

The data sets used and/or analyzed during the current study are available from the corresponding author on request.

\section{Competing interests}

The authors have no competing interests to declare.

\section{Consent for publication}

Not applicable. The manuscript does not contain patient identifiable data.

\section{Ethics Approval and consent to participate}

Ethical approval was sought and granted by two organizations. One from the university board of the British University in Dubai and another approval was taken from the Research and Ethical (REC) committee from Ministry and Health and Prevention by applying to them and filling their ethical approval form. Written Informed consent was also obtained.

\section{Funding}

This research received no specific grant from any funding agency in the public, commercial, or not-for-profit sectors. 
Authors' contributions

$\mathrm{AH}$ was involved in all stages of the research process including data collection, analysis, acquisition and writing.

\section{Acknowledgements}

I would like to thank Ministry of Health and Prevention, UAE for the constant support and encouragement.

\section{References}

1. Khoja S, Scott RE, Casebeer AL, Mohsin M, Ishaq AF, Gilani S. e-Health readiness assessment tools for healthcare institutions in developing countries. Telemedicine and e-Health. 2007 Aug 1;13(4):425-32.

2. Fernández-Alemán JL, Señor IC, Lozoya PÁ, Toval A. Security and privacy in electronic health records: A systematic literature review. Journal of biomedical informatics. 2013 Jun 1;46(3):541-62.

3. Hasanain RA, Cooper H. Solutions to overcome technical and social barriers to electronic health records implementation in Saudi public and private hospitals. Journal of Healthlnformatics in Developing Countries. 2014 May 12;8(1).

4. Biruk S, Yilma T, Andualem M, Tilahun B. Health Professionals' readiness to implement electronic medical record system at three hospitals in Ethiopia: a cross sectional study. BMC medical informatics and decision making. 2014 Dec;14(1):115.

5. Willyard C. Electronic records pose dilemma in developing countries. 2010. Nat Med;16:249.

6. Ventures IH. Medical Center Electronic Health Record Readiness Assessment. InTech Health Ventures. 2008.

7. Khoja S, Scott RE, Casebeer AL, Mohsin M, Ishaq AF, Gilani S. e-Health readiness assessment tools for healthcare institutions in developing countries. Telemedicine and e-Health. 2007 Aug 1;13(4):425-32.

8. Ajami S, Ketabi S, Isfahani SS, Heidari A. Readiness assessment of electronic health records implementation. Acta Informatica Medica. 2011 Dec;19(4):224.

9. World Health Organization. Electronic Health Records: Manual for Developing Countries. Manila, Philippines: WHO Regional Office for the Western Pacific. 2006.

10. Kuo KM, Liu CF, Ma CC. An investigation of the effect of nurses' technology readiness on the acceptance of mobile electronic medical record systems. BMC Med Inform Decis Mak,2013. 13(1):88. doi: 10.1186/1472-6947-13-88.

11. Al-Aswad AM, Brownsell S, Palmer R, Nichol JP. A review paper of the current status of electronic health records adoption worldwide: the gap between developed and developing countries. $J$ Health Inform Dev Ctries; 2013; 7(2):153-164.

12. Anwar F, Shamim A, Khan S. Barriers in adoption of health information technology in developing societies. Int J Adv Comput Sci Appl; 2011: 2:40-45.

13. Gamm LD, Barsukiewicz CK, Dansky KH, Vasey JJ, Bisordi JE, Thompson PC. Proceedings of the AMIA Symposium. Pennsylvania, United States: American Medical Informatics Association;. Pre- and post- 
control model research on end-users' satisfaction with an electronic medical record: preliminary results. 1998.

14. Braun, V., \& Clarke, V. Using thematic analysis in psychology. Qualitative research in psychology, 2006: 3(2), 77-101.

15. Bramble, J. D., Galt, K. A., Siracuse, M. V., Abbott, A. A., Drincic, A., Paschal, K. A., \& Fuji, K. T. The relationship between physician practice characteristics and physician adoption of electronic health records. Health care management review, 2010; 35(1), 55-64.

16. El-Hassan, O., Sharif, A., Al, M. R., \& Blair, I. Tracking the Implementation of Electronic Medical Records in Dubai, United Arab Emirates, Using an Adoption Benchmarking Tool. Studies in health technology and informatics; 2017: 245, 64-68.

17. Simon, J. S., Rundall, T. G., \& Shortell, S. M. Drivers of electronic medical record adoption among medical groups. The Joint Commission Journal on Quality and Patient Safety, 2005: 31(11), 631-639.

18. Snyder, R. A., \& Fields, W. L. Measuring hospital readiness for information technology (IT) innovation: a multisite study of the organizational information technology innovation readiness scale. $J$ Nurs Meas, 2006: 14(1), 45.doi: 10.1891/jnum.14.1.45.

19. Zandieh, S. O., Yoon-Flannery, K., Kuperman, G. J., Langsam, D. J., Hyman, D., \& Kaushal, R. Challenges to EHR implementation in electronic-versus paper-based office practices. Journal of general internal medicine, 2008: 23(6), 755-761.

20. DesRoches, C. M., Campbell, E. G., Rao, S. R., Donelan, K., Ferris, T. G., Jha, A. \& Blumenthal, D. Electronic health records in ambulatory care-a national survey of physicians. N Engl J Med,2008: 359(1), 50-60.

21. Accenture. (2010). Overview of international EMR/EHR markets; retrieved from https://www.accenture.com/usen/ /media/Accenture/ConversionAssets/DotCom/Documents/Global/PDF/Industries_11/AccentureGetting-EMR-Back-Fast-Lane.pdf. (Accessed on 10 ${ }^{\text {th }}$ June 2019).

22. Ferranti, J. M., Musser, R. C., Kawamoto, K., \& Hammond, W. E. The clinical document architecture and the continuity of care record: a critical analysis. Journal of the American Medical Informatics Association; 2006: 13(3), 245-252.

23. Rae, A., \& Nasser, R. Managing Development for the Health Records to be centralized electronically, in UAE (Doctoral dissertation, The British University in Dubai (BUiD)). 2011.

24. Pearce CM, De Lusignan S, Phillips C, Hall S, Travaglia J. The computerized medical record as a tool for clinical governance in Australian primary care. Interactive journal of medical research. 2013;2(2):e26.

25. Drew, D. Public-Private inter-organizational sharing of health data for disability determination (Doctoral dissertation). 2011.

26. MOHAP. National Unified Medical Records (NUMR) Vision \& Strategy. Ministry of health and prevention. 2017 